\title{
Evans Stage III
}

National Cancer Institute

\section{Source}

National Cancer Institute. Evans Stage III. NCI Thesaurus. Code C85410.

Tumor spread in continuity beyond the midline or tumor spread to lymph nodes bilaterally. 